\title{
Power Allocation Approaches for Combined Positioning and Communications OFDM Systems
}

\author{
Rafael Montalban, José A. López-Salcedo and Gonzalo Seco-Granados \\ Universitat Autònoma de Barcelona, \\ Bellaterra, Barcelona 08193, Spain \\ \{rafael.montalban, jose.salcedo, gonzalo.seco\}@uab.cat
}

\author{
A. Lee Swindlehurst \\ Univ. of California, Irvine, \\ Irvine, CA 92697, USA \\ swindle@uci.edu
}

\begin{abstract}
The design of pilot and data power allocations for multicarrier OFDM signals is a key aspect in the development of combined positioning and high-data-rate communications systems. Previous work has investigated the capacity-maximizing pilot and data power allocation when only taking into account the effect of channel estimation accuracy on capacity. Results have shown that distributions with equi-spaced and equi-powered pilot structures are optimal. In this paper, we consider a formulation based on the CRB of the joint time-delay and channel estimation, which allows us to study the design of signals for combined positioning and communications OFDM systems. We compare the performance of capacity-maximizing pilot and data power distributions with unstructured pilots, with respect to distributions that use equi-spaced and equi-powered pilot structures. Numerical results show that the constraint of equi-spaced and equi-powered pilot structures has an important impact on both the achievable capacity and the positioning capabilities of the designed signals.
\end{abstract}

\section{INTRODUCTION}

There exists an increasing interest in combined positioning and communications systems, as evidenced by the efforts to use or adapt multicarrier communications signals for positioning applications [1], [2]. However, the signals designed for communications can achieve very high data rates (tens to hundreds of Mbits per second), but are not adequate in general for accurate positioning. A proof of this is that the latest generation communications standards include signal configurations specifically targeted for positioning, such as LTE's (Long Term Evolution) positioning reference signal (PRS) [3]. Nevertheless, the design of combined positioning and communications systems that can perform well in both domains is a topic that deserves further study, and needs to be formulated taking into account all the parameters that affect both the system's achievable data rate and positioning accuracy.

Optimal pilot design for channel estimation has been studied extensively in the literature for orthogonal frequency division multiplexing (OFDM) signals [4]-[7]. Results show that equi-spaced (in a circular sense) and equi-powered pilots are optimal in terms of channel estimation mean square error. Therefore, these kinds of pilot distributions appear to be the option chosen by most communications standards. Likewise, the design of signals for estimation of carrier frequency offset (CFO) and for joint channel and CFO estimation has been

This work was partly supported by the Spanish Government under grant TEC2011-28219 and by the Catalan Government under grant 2009 SGR 298. considered in the literature, and optimal training signals have been proposed [8], [9].

As wireless multicarrier systems rely on pilot symbols for channel estimation, it seems reasonable to apply these pilot symbols for time-delay estimation (TDE) as well, which is the basis for positioning. Several researchers have studied the problem of achieving high time-delay estimation accuracy in multicarrier systems. In [10], [11], it was shown that for a pilot-only multicarrier signal (i.e. when any subcarrier can be used to transmit pilot symbols), minimizing the variance of the time-delay estimate requires maximizing the root-meansquare (or Gabor) bandwidth of the signal. In [12], the problem of pilot design is considered for combined time-delay and channel estimation in OFDM signals. The design requires a trade-off between both estimation accuracies, but in general the optimal structures consist of pilot subcarriers at the edges of the bandwidth and approximately equi-spaced pilot subcarriers in between. In [13], the problem of finding the capacitymaximizing pilot and data power distributions for OFDM systems, while taking into account the capabilities of the designed signal for positioning, is studied. Moreover, a nearly optimal power allocation approach is presented as a solution.

This paper studies the performance of equi-powered and equi-spaced pilot allocation for joint time-delay and channel estimation, and compares it with the performance of optimal arbitrary allocations obtained in [13]. We focus on a scenario where a certain positioning capability is desired (i.e., we fix a desired time-delay estimation accuracy), and the system is designed to achieve the highest possible capacity, while guaranteeing this positioning accuracy at the same time. Our results show that restricting the pilot distributions to be equipowered and equi-spaced produces a noticeable degradation on the maximum achievable capacity and TDE accuracy.

The paper is organized a follows. Section II presents the system model and performance metrics. Section III reviews the problem of capacity maximization under a constraint on the time-delay estimation accuracy and dwells on the special case where only equi-spaced and equi-powered pilot distributions are considered. Simulations and numerical examples are presented in Section IV, before drawing the conclusions in Section V.

\section{SYSTEM MODEL AND PRELIMINARIES}

In this section we describe the signal model and the performance metrics used for the design of the OFDM pilot 
and data power distributions. We use two performance metrics: an approximation on the channel capacity when there is uncertainty in the channel state information, and the Cramér-Rao bound (CRB) of the joint time-delay and channel estimates.

\section{A. OFDM Signal Model}

Consider the following frequency-selective channel model,

$$
h(t)=\sum_{l=0}^{L-1} h_{l} \delta\left(t-l T_{s}-\tau_{d}\right)
$$

where $L$ is an upper bound on the number of discrete multipath components, $h_{l}$ is the complex channel gain for the $l$-th path, $T_{s}$ is the sampling period and $\tau_{d}$ is the time-delay between source and receiver. Note that since the delay is explicitly modeled inside the terms $\delta\left(t-l T_{s}-\tau_{d}\right)$, the channel coefficients $\left\{h_{l}\right\}$ are independent of $\tau_{d}$.

In our case, we assume that all information about the channel is unknown, so both the channel coefficients $\left\{h_{l}\right\}$ and the channel delay $\tau_{d}$ need to be estimated through the use of pilot tones transmitted as part of the $N$ subcarriers in an OFDM symbol. For the scope of this paper, we restrict our problem to the zero inter-carrier and inter-symbol interference case. This means that the transmitter and receiver are frequency synchronized, and that the duration of the cyclic prefix (CP) $T_{G}$ is larger than the delay spread plus the time-delay uncertainty (i.e. $\left.(L-1) T_{s}+\tau_{d}<T_{G}\right)$.

Consider now the vector containing the discrete Fourier transform (DFT) of $N$ samples of the received signal collected during an OFDM symbol,

$$
\mathbf{y}=\boldsymbol{\Gamma}\left(\tau_{d}\right) \mathbf{S}(\mathbf{s}) \mathbf{W}_{L} \mathbf{h}+\mathbf{n},
$$

where [12]

$$
\begin{aligned}
\boldsymbol{\Gamma}\left(\tau_{d}\right)= & \operatorname{diag}\left(\left[e^{-j 2 \pi \frac{-N / 2+1}{T_{s}} \tau_{d}}, e^{-j 2 \pi \frac{-N / 2+2}{T_{s}} \tau_{d}},\right.\right. \\
& \left.\left.\ldots, e^{-j 2 \pi \frac{N / 2}{T_{s}} \tau_{d}}\right]\right) \\
\mathbf{s}= & {\left[b_{-N / 2+1}, b_{-N / 2+2}, \ldots, b_{N / 2}\right]^{T}, } \\
\mathbf{S}(\mathbf{s})= & \operatorname{diag}(\mathbf{s}), \\
\mathbf{h}= & {\left[h_{0}, h_{1}, \ldots, h_{L-1}\right]^{T}, }
\end{aligned}
$$

$\mathbf{W}_{L}$ is composed of the first $L$ columns of the zero-frequency centered $N \times N$ Fourier matrix, $\mathbf{n}$ is a vector of additive Gaussian noise, and s contains the both the pilot and the data symbols being transmitted.

\section{B. The Cramér-Rao Bound}

For the derivation of the $\mathrm{CRB}$, we ignore the presence of data symbols for the purpose of estimation, and assume that the vector s contains pilot symbols only. We define the following parameter vector,

$$
\boldsymbol{\Theta}:=\left[\tau_{d}, \Re\left\{\mathbf{h}^{T}\right\}, \Im\left\{\mathbf{h}^{T}\right\}\right] \in \mathbb{R}^{(2 L+1) \times 1},
$$

which contains the time-delay and the real $\left(\Re\left\{\mathbf{h}^{T}\right\}\right)$ and imaginary ( $\Im\left\{\mathbf{h}^{T}\right\}$ ) parts of the channel response.

As shown in [12], the expression for the CRB of $\Theta$ is:

$$
\mathbf{C R B}_{\Theta}=\frac{\sigma_{n}^{2}}{2}\left[\begin{array}{cc}
\mathrm{CRB}_{11} & \mathbf{C R B}_{21}^{T} \\
\mathbf{C R B}_{21} & \mathbf{C R B}_{22}
\end{array}\right]
$$

where

$$
\begin{aligned}
& \mathrm{CRB}_{11}^{-1}=\mathbf{h}^{H} \mathbf{W}_{L}^{H} \mathbf{P D}^{2} \mathbf{W}_{L} \mathbf{h} \\
& -\mathbf{h}^{H} \mathbf{W}_{L}^{H} \mathbf{P D} \mathbf{W}_{\mathbf{L}}(\mathbf{Q})^{-1} \mathbf{W}_{L}^{H} \mathbf{D P} \mathbf{W}_{L} \mathbf{h}, \\
& \mathbf{C R B}_{21}=\left[\begin{array}{c}
-\gamma_{\tau}^{-1} \Im\{\mathbf{q}\} \\
\gamma_{\tau}^{-1} \Re\{\mathbf{q}\}
\end{array}\right] \text {, } \\
& \mathbf{C R B}_{22}=\left[\begin{array}{c}
\Re\left\{\mathbf{Q}^{-1}\right\}+\gamma_{\tau}^{-1} \Im\{\mathbf{q}\} \Im\left\{\begin{array}{l}
\mathbf{q}^{T} \\
\Im \\
\mathbf{Q}^{-1}
\end{array}\right\}-\gamma_{\tau}^{-1} \Re\{\mathbf{q}\} \Im\left\{\mathbf{q}^{T}\right.
\end{array}\right\} \\
& \left.\begin{array}{l}
-\Im\left\{\mathbf{Q}^{-1}\right\}-\gamma_{\tau}^{-1} \Im\{\mathbf{q}\} \Re\left\{\mathbf{q}^{T}\right\} \\
\Re\left\{\mathbf{Q}^{-1}\right\}+\gamma_{\tau}^{-1} \Re\{\mathbf{q}\} \Re\left\{\mathbf{q}^{T}\right\}
\end{array}\right], \\
& \mathbf{q}=\mathbf{Q}^{-1} \mathbf{W}_{L}^{H} \mathbf{P D} \mathbf{W}_{L} \mathbf{h}, \\
& \mathbf{Q}=\mathbf{W}_{L}^{H} \mathbf{P} \mathbf{W}_{L} \text {, } \\
& \mathbf{D}=\frac{2 \pi}{T_{s}} \operatorname{diag}\left(\left[-\frac{N}{2}+1, \ldots, \frac{N}{2}\right]\right), \\
& \mathbf{P}=\operatorname{diag}\left(\mathbf{p}_{p}\right)=\mathbf{S}^{H} \mathbf{S},
\end{aligned}
$$

where vector $\mathbf{p}_{p}$ contains the power of the pilots assigned to each subcarrier. Note that the CRB only depends on the amount of power assigned to each of the pilot subcarriers.

\section{Capacity}

In [14] and [15] a lower bound on the channel capacity for serial transmissions over flat-fading channels was derived. We apply this lower bound on a per-subcarrier basis, and summing across the data subcarriers, we have the following lower bound on the multicarrier signal channel capacity:

$$
C \geq C_{l b}=\frac{1}{N} \sum_{i \in \Omega} \log \left(1+\mathrm{SNReq}_{i}\right)
$$

where $\Omega$ contains the indices of the subcarriers allocated to data transmission, and where an equivalent signal to noise ratio for subcarrier $i$ is defined as,

$$
\mathrm{SNReq}_{i}=\frac{\left|g_{i}\right|^{2} p_{d, i}}{\sigma_{g, i}^{2} p_{d, i}+\sigma_{n}^{2}},
$$

where $g_{i}$ is the channel frequency response for subcarrier $i$, $p_{d, i}$ is the power allocated to data transmission in subcarrier $i$, $\sigma_{g, i}^{2}$ is the variance of the channel response estimate at the $i$-th subcarrier and $\sigma_{n}^{2}$ is the Gaussian noise power. The inequality in (16) becomes an equality only when channel estimation uncertainty has the worst possible effect [15].

For an unbiased estimator, the value of $\sigma_{g_{i}}^{2}$ is lower bounded by the CRB of the channel frequency response estimate for subcarrier $i$, which can be computed from (11) as:

$$
\mathrm{CRB}_{\mathrm{g}}=\mathbf{T C R B}_{\mathbf{h}} \mathbf{T}^{H}
$$

where

$$
\mathbf{T}=\left[\begin{array}{ccc}
\Re\left\{\mathbf{W}_{L}\right\} & -\Im & \left.\mathbf{W}_{L}\right\} \\
\Im\left\{\mathbf{W}_{L}\right\} & \Re & \left.\mathbf{W}_{L}\right\}
\end{array}\right]
$$

In this paper, we consider the maximization of the lower bound on the capacity assuming that the lowest possible uncertainty in the channel estimates is achieved. Thus, the approximation we will use is

$$
C_{l b}^{\prime}=\frac{1}{N} \sum_{i \in \Omega} \log \left(1+\frac{\left|g_{i}\right|^{2} p_{d, i}}{\left[\mathbf{C R B}_{g}\right]_{i i} p_{d, i}+\sigma_{n}^{2}}\right)
$$




\section{Power Allocation Optimization}

\section{A. Unstructured Pilot Distributions}

In order to maximize the capacity in (20) while ensuring a certain level of positioning capabilities (i.e. a certain level of time-delay estimation accuracy), we need to adequately distribute pilot and data power across the subcarriers.

A possible formulation of the problem is

$$
\begin{cases}\max _{\mathbf{p}_{p}, \mathbf{p}_{d}} & C_{l b}^{\prime}\left(\mathbf{p}_{p}, \mathbf{p}_{d}\right) \\ \text { s.t. } & \mathrm{CRB}_{11}\left(\mathbf{p}_{p}\right) \leq \beta \\ & \mathbf{p}_{p}^{T} \cdot \mathbf{1}+\mathbf{p}_{d}^{T} \cdot \mathbf{1} \leq P_{T} \\ & \mathbf{p}_{p}^{T} \cdot \mathbf{p}_{d}=0 \\ & \mathbf{p}_{p} \geq \mathbf{0} \\ & \mathbf{p}_{d} \geq \mathbf{0}\end{cases}
$$

where $\mathbf{p}_{p}$ is a vector that contains the powers assigned to pilot symbols in each subcarrier, $\mathbf{p}_{d}$ contains the power of subcarriers devoted to data transmission and $P_{T}$ is the total available power. The first constraint in (21) ensures that the $\mathrm{CRB}$ of the time-delay estimation is lower than a certain value $\beta$. The third restriction in (21) causes the problem to be combinatorial, i.e., the subcarriers are going to be assigned to either pilot or data symbols.

Alternatively, the problem may be written as

$$
\left\{\begin{array}{cl}
\underset{\mathbf{p}, \mathbf{b}}{\max } & C_{l b}^{\prime}(\mathbf{p}, \mathbf{b}) \\
\text { s.t. } & \mathrm{CRB}_{11}(\mathbf{p}, \mathbf{b}) \leq \beta \\
& \mathbf{p}^{T} \cdot \mathbf{1} \leq P_{T} \\
& \mathbf{p} \geq \mathbf{0} \\
& b_{i}^{2}-b_{i}=0
\end{array}\right.
$$

where vector $\mathbf{p}$ contains the powers assigned to each of the subcarriers (i.e. $\mathbf{p}=\mathbf{p}_{p}+\mathbf{p}_{d}$ ), and $\mathbf{b}$ is a binary vector of length $N$ than contains a ' 1 ' in the position corresponding to pilot subcarriers and a ' 0 ' in the positions corresponding to data subcarriers. Thus, the elements of vector $\mathbf{p}_{p}$ can be expressed as $p_{p, i}=b_{i} p_{i}$ and the elements of vector $\mathbf{p}_{d}$ as $p_{p, i}=p_{i}\left(1-b_{i}\right)$, where $b_{i}$ is the $i$-th element of vector $\mathbf{b}$.

In [13], an almost optimal solution to this problem was studied. The problem was solved through the use of a relaxed version that eliminated the combinatorial constraint. That is, if we relax the third restriction in (21) (i.e. the fourth restriction in (22)), the subcarriers can be shared by data and pilot symbols simultaneously. The solution to this relaxed problem can be found numerically by using standard convex programming approaches which are guaranteed to converge to an optimal solution. Simulation results show that, in the solutions to the relaxed problem, the number of subcarriers with $p_{p, i}>0$ is very low, generally equal or close to $L+1$, which is the lowest value required for the problem to be identifiable. In terms of the formulation presented in (22), we can see that optimal solutions for the relaxed problem consist of $\mathbf{a}$ vector $\mathbf{b}$ with most of its entries set to ' 0 ' and just a few set to values greater than ' 0 '.

After solving the relaxed problem, one could approximate the almost-binary vector $\mathbf{b}$ by a completely binary vector. The maximization problem in (22) can then be easily solved when the subcarrier assignment (i.e., the values of $b_{i}$ ) are considered as fixed. As discussed in [13], the resulting power distribution is close or equal to the optimum solution of (21).

\section{B. Equi-spaced and Equi-powered Pilot Distributions}

For comparison purposes, we consider now the case where we restrict ourselves to use equi-powered and equi-spaced pilot distributions only. For the sake of brevity, we also refer to this kind of pilot distributions as "comb" distributions in the sequel. We consider a pilot distribution that uses $K$ out of the $N$ tones in an OFDM symbol. Equi-spaced (in circular sense) distributions can only exist if $K$ is a divisor of $N$. Thus, $N$ must be a multiple of $K$ such that $N=K J$, for some integer $J$. The set of possible pilot positions for an equispaced distribution is $\mathcal{I}=\{j+J k \mid k \in[0, K-1]\}$ for some $j \in[0, J-1]$.

Note that $K$ must be at least $L+1$ for the estimation problem to be feasible. Apparently, from the point of view of capacity we would like to use the minimum number of pilots, i.e. $K=L+1$, in order to have as many subcarriers free for data transmission as possible. However, depending on the values of $N$ and $L$, this may be impossible. In the sequel we will assume that $N$ is a multiple of $L+1$ and $K=L+1$, to focus our analysis on the effect of employing comb pilot distributions instead of unstructured ones, and not be affected by the fact that in general $K$ has to be larger than $L+1$ to be a divisor of $N$.

The capacity maximization problem we consider in this case is:

$$
\begin{cases}\max _{P_{p}, \mathbf{p}_{d}, j} & C_{l b}^{\prime}\left(\mathbf{p}_{p}\left(P_{p}, j\right), \mathbf{p}_{d}\right) \\ \text { s.t. } & \mathrm{CRB}_{11}\left(\mathbf{p}_{p}\left(P_{p}, j\right)\right) \leq \beta \\ & P_{p}+\mathbf{p}_{d}^{T} \cdot \mathbf{1} \leq P_{T} \\ & \mathbf{p}_{p}\left(P_{p}, j\right)^{T} \cdot \mathbf{p}_{d}=0 \\ & \mathbf{p}_{d} \geq \mathbf{0}\end{cases}
$$

where the pilot distribution $\mathbf{p}_{p}$ is indeed forced to be equispaced and equi-powered (this is denoted as $\mathbf{p}_{p}\left(P_{p}, j\right)$ ), and thus is a function of the total pilot power $P_{p}$ and $j$. This problem can be easily solved numerically.

It is also interesting to consider a related problem where the amount of power for pilot transmission $P_{p}$ is fixed and there is no restriction on the CRB of the time-delay estimate. This allows for comparisons of how the capacity and the time-delay estimation accuracy change with respect to those achieved by an unstructured pilot power distribution that devotes the same amount of power to pilot and data transmission. The resulting problem is similar to (23), without the first constraint and with $P_{p}$ now fixed to a given value.

\section{PERFormance ANALYSis}

In this section we compute several pilot and data power distributions obtained as a solution to the optimization problems discussed in Section III. For all the results presented, we assume a channel impulse response of length $L=7$, with $\mathbf{h}=[-0.4487-i 0.4070,-2.1060+i 0.6322,-0.1651-$ $i 1.1886,0.5449-i 0.1982,0.2716+i 0.2939,-0.3408+$ $i 0.7404,1.0934+i 0.0397]^{T}$, and an OFDM signal of $N=64$ subcarriers. The total power for pilots plus data has been fixed 
to $P_{T}=5$, the noise variance to $\sigma_{n}^{2}=10^{-2}$ and the maximum value of $\mathrm{CRB}_{11}$ has been constrained to be $\beta=0.001$.

Figure 1 shows the data and power distributions designed to optimize the lower bound on capacity $C_{l b}^{\prime}$, obtained under different considerations. Fig. 1a shows an example of pilot and data power distribution obtained fixing the subcarrier positions after the problem relaxation, as described in Section III.A (i.e. a solution to (21)). Note that pilot power seems to be more or less evenly distributed among the pilot subcarriers, while data power assignment is a water-filling distribution. Fig. 1b shows the optimal power allocation with the comb pilot structure that achieves the same time-delay estimation accuracy as the distribution in Fig. 1a (i.e., a solution to (23)). Note that the amount of pilot power needed to fulfill the constraint is much greater if we restrict ourselves to use comb distributions, which has a negative impact on the capacity. Finally, Fig. 1c shows the optimal power distribution obtained when restricting the pilots to be a comb structure and the amount of pilot power to be the same as the distribution in Fig. 1a. In this case, while the capacity is more or less the same as in the unstructured-pilots case, the time-delay estimation accuracy significantly worsens (i.e., it leads to an increase in the CRB). Note that the pilots are similarly distributed in both Fig. 1a and Fig. 1b, and the amount of power used is the same, however the effect of the restriction to a comb pilot distribution has a dramatic negative impact on the CRB.

Figure 2 studies the trade-off between capacity and timedelay estimation uncertainty. The curve with square markers represents the capacity achieved by distributions obtained through the maximization of $C_{l b}^{\prime}$ without any restriction on the pilot distributions that may be used, i.e. solutions to (21) for differents values of $\beta$ obtained through the process of fixing the subcarrier positions after the problem relaxation, as described in Section III.A. The curve with circle markers corresponds to the case of distributions designed with comb pilot structures that fulfill the constraint on time-delay accuracy, i.e. solutions to (23). Note that in any case where the total available power is not enough for a comb pilot structure to fulfill the constraint, the capacity is 0 since there is no power left for data transmission. The curve with point markers corresponds to distributions with comb pilot structures that use the same total power for pilot transmission as in the unstructured-pilots case. It is very important to note that in this case the distributions do not achieve the desired CRB and the percentage by which they exceed the constraint is represented in the dash-dot curve. The capacity achieved by optimal power distributions when subcarriers are allowed to be shared by pilot and data symbols, i.e. solutions to the relaxed version of (21), is provided as an upper bound of the maximum achievable capacity (solid line with no markers).

Figure 3 shows the amount of extra power required by a distribution with comb pilot structure to match the performance of a distribution with unstructured pilots. The curve with square markers represents the extra power needed to achieve the same TDE accuracy (i.e. the same CRB), while the curve with point markers represents the additional power needed to achieve the same capacity and TDE accuracy. That is, the second curve shows how much $P_{T}$ has to be increased in (23) with respect to the value used in (21) so that both problems have the same value of the $C_{l b}^{\prime}$ for each value of $\beta$. This

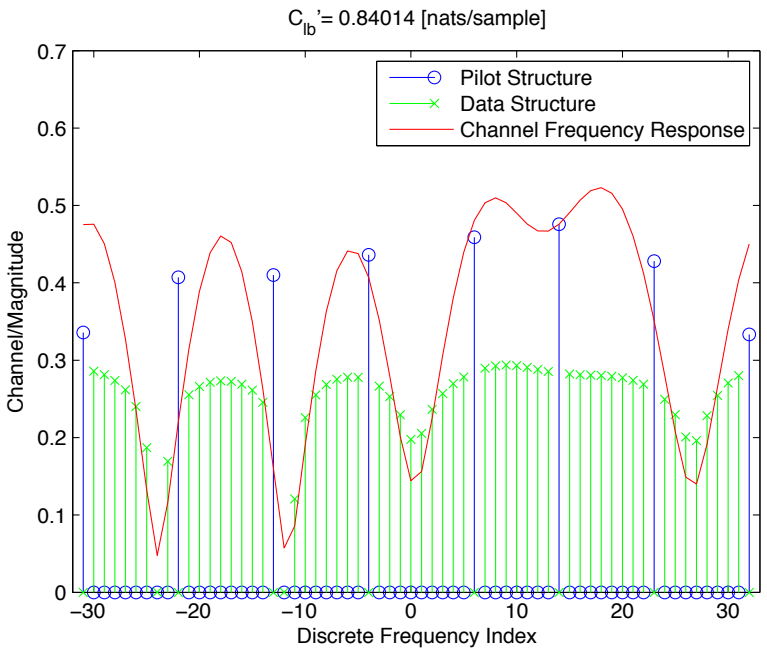

(a)

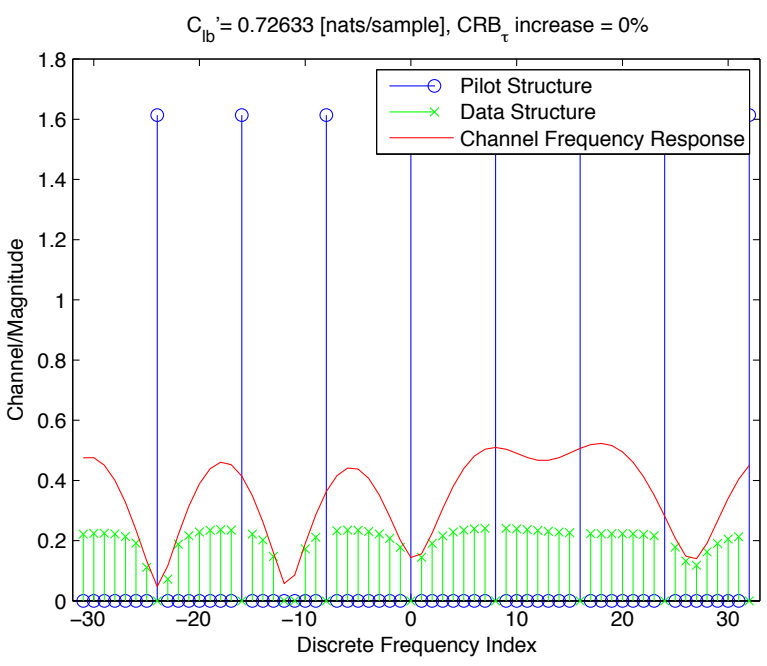

(b)

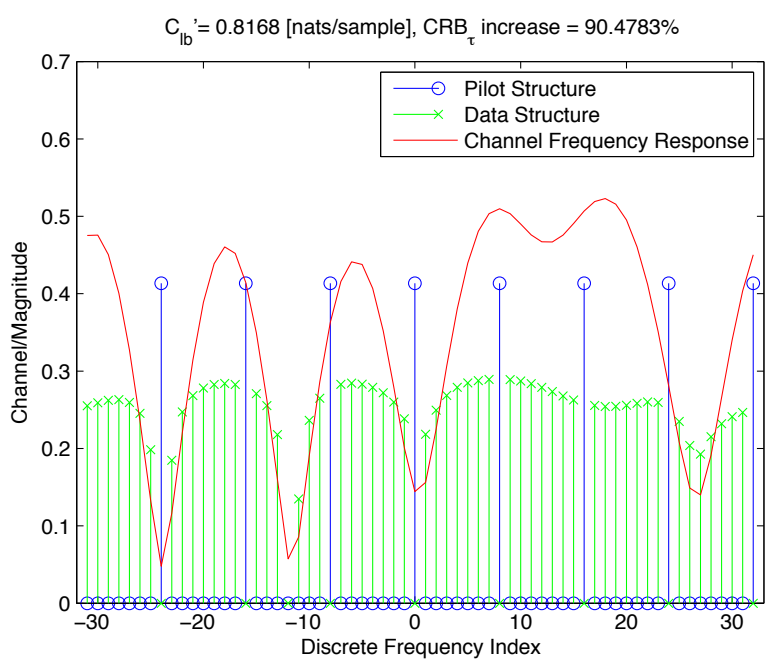

(c)

Fig. 1. Pilot and data power distributions designed to maximize $C_{l b}^{\prime}$ under different conditions: (a) Unstructured pilots. (b) Equi-spaced and equi-powered pilots, same time-delay estimation accuracy. (c) Equi-spaced and equi-powered pilots, same total power for pilot transmission as in case (a). 


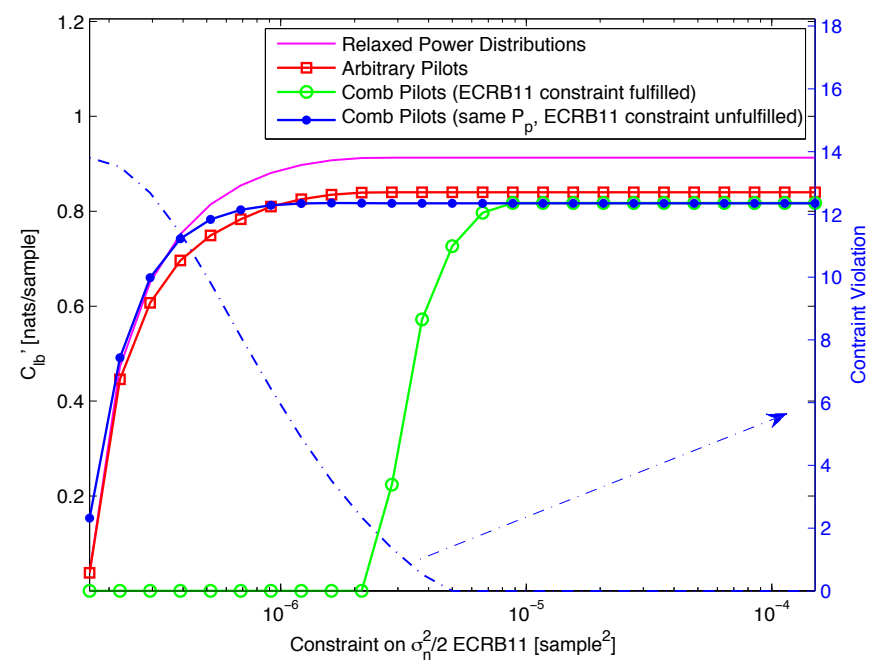

Fig. 2. Channel capacity versus constraint on time-delay estimation accuracy.

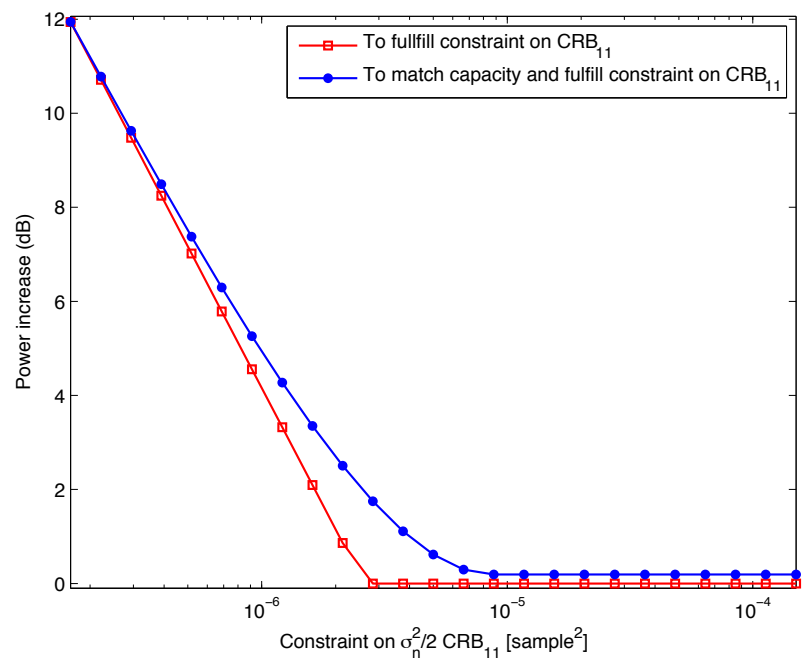

Fig. 3. Increase in total power required to match the performance of an unstructured distribution with an equi-spaced and equipowered distribution, versus the contraint on time-delay estimation accuracy.

justifies the use of unstructured pilot distributions for combined communications and positioning systems.

Results show that for a fixed amount of power devoted to pilot transmission, a distribution with comb pilots obtains similar results in terms of capacity as an unstructured distribution (as reflected by the solid lines with point and square markers in Fig. 2). This means that comb pilots are adequate only when one is designing a communications system, which agrees with previous results in the literature and with the use of this type of pilot structure in the communications standards. However, our results clearly show that comb pilot distributions are inadequate when the positioning capabilities of the signal are a concern. In order to match the performance of an unstructured distribution, comb distributions require much more power.

\section{Conclusions}

In this paper, the performance of near-optimal pilot and data power allocations has been compared with the tradi- tional solution of using equi-powered and equi-spaced pilot distributions. Results show that equi-spaced and equi-powered pilot structures are not adequate for time-delay estimation as they require important increases in the amount of power devoted to pilot transmission in order to achieve the same time-delay estimation accuracy as power distributions with unstructured pilots, which results in lower capacity, since less power remains available for data. Equi-spaced and equipowered pilot distributions are only a good choice when designing a communications-only system, where time-delay estimation accuracy is not an objective by itself.

\section{REFERENCES}

[1] F. Gustafsson and F. Gunnarsson, "Mobile positioning using wireless networks: possibilities and fundamental limitations based on available wireless network measurements," Signal Processing Magazine, IEEE, vol. 22, pp. 41-53, july 2005 .

[2] V. Moghtadaiee, S. Lim, and A. Dempster, "System-level considerations for signal-of-opportunity positioning," in Int. Symp. GPS/GNSS, Taipei, Taiwan, 2010.

[3] J. del Peral-Rosado, J. Lopez-Salcedo, G. Seco-Granados, F. Zanier, and M. Crisci, "Achievable localization accuracy of the positioning reference signal of 3GPP LTE," in Proc. International Conference on Localization and GNSS (ICL-GNSS). IEEE, 2012.

[4] I. Barhumi, G. Leus, and M. Moonen, "Optimal training design for MIMO OFDM systems in mobile wireless channels," Signal Processing, IEEE Transactions on, vol. 51, no. 6, pp. 1615-1624, 2003.

[5] S. Ohno and G. Giannakis, "Capacity maximizing MMSE-optimal pilots for wireless OFDM over frequency-selective block rayleighfading channels," IEEE Transactions on Information Theory, vol. 50, no. 9 , pp. 2138 - 2145, sept. 2004.

[6] D. Hu, L. Yang, Y. Shi, and L. He, "Optimal pilot sequence design for channel estimation in MIMO OFDM systems," Communications Letters, IEEE, vol. 10, no. 1, pp. 1-3, 2006.

[7] S. Ohno, E. Manasseh, and M. Nakamoto, "Preamble and pilot symbol design for channel estimation in OFDM systems with null subcarriers," EURASIP Journal on Wireless Communications and Networking, vol. 2011, no. 1, pp. 1-17, 2011.

[8] H. Minn and S. Xing, "An optimal training signal structure for frequency-offset estimation," Communications, IEEE Transactions on, vol. 53, no. 2, pp. 343-355, 2005.

[9] H. Minn, N. Al-Dhahir, and Y. Li, "Optimal training signals for MIMO OFDM channel estimation in the presence of frequency offset and phase noise," Communications, IEEE Transactions on, vol. 54, no. 10, pp. 1754-1759, 2006.

[10] F. Zanier and M. Luise, "Fundamental issues in time-delay estimation of multicarrier signals with applications to next-generation GNSS," in 10th International Workshop on Signal Processing for Space Coтmunications SPSC 2008, 2008, pp. 1-8.

[11] _ "A new look into the issue of the Cramér-Rao Bound for delay estimation of digitally modulated signals," in Proceedings of the 2009 IEEE International Conference on Acoustics, Speech and Signal Processing, 2009, pp. 3313-3316.

[12] M. Larsen, G. Seco-Granados, and A. Swindlehurst, "Pilot optimization for time-delay and channel estimation in OFDM systems," in Acoustics, Speech and Signal Processing (ICASSP), 2011 IEEE International Conference on. IEEE, 2011, pp. 3564-3567.

[13] R. Montalban, G. Seco-Granados, and A. Swindlehurst, "Suboptimal method for pilot and data power allocation in combined positioning and communications OFDM systems," in 2012 Asilomar Conference on Signals, Systems, and Computers, 2012.

[14] E. Biglieri, J. Proakis, and S. Shamai, "Fading channels: Informationtheoretic and communications aspects," Information Theory, IEEE Transactions on, vol. 44, no. 6, pp. 2619-2692, 1998.

[15] M. Medard, "The effect upon channel capacity in wireless communications of perfect and imperfect knowledge of the channel," Information Theory, IEEE Transactions on, vol. 46, no. 3, pp. 933-946, 2000. 